\title{
Colour Gradients in Galaxy Bulges
}

\author{
MARC BALCELLS \\ Kapteyn Lab, Postbus 800, 9700 AV Groningen, Netherland \\ and \\ R. F. PELETIER \\ E.S.O., Karl Schwarzschildstrasse, 2, D-8046 Garching bei München, Germany
}

November 27,1992

\section{Introduction}

Colour gradients, when due to stellar populations rather than dust, are potential diagnostics on the formation history of galaxy bulges. We have carried out measurements of colour gradients on a sample of 30 bulges, the first such study using CCD detectors.

We have looked for two types of dependencies: 1) with total and bulge luminosities; 2) with disk parameters, such as central surface brightness and disk colours. We also compare bulges' gradients to colour gradients in elliptical galaxies.

\section{Data, analysis}

Our sample comprises galaxies of types $\mathrm{S}, \mathrm{S} 0-\mathrm{Sb}$ from the UGC catalog with $B_{T} \leq$ 14 , with diameters above 2' and axial ratios above 2.1. Barred galaxies have been excluded.

Images in $\mathrm{U}, \mathrm{B}, \mathrm{R}, \mathrm{I}$ were obtained at the prime focus of the Isaac Newton Telescope with a GEC C $\dot{C}$. Conditions were photometric, but there wa some Sahara dust. Flat fielding was always better than $0.4 \%$. Uncertainties in the absolute photometry were $0.05 \mathrm{mag}$ in $\mathrm{R}$ and $\mathrm{I}$, and $0.1 \mathrm{mag}$ in $\mathrm{U}$ and B. Bulge-disk decomposition was carried out following the scheme used by Kent (1986).

Identifying the effects of dust is the main difficulty of this project. Galaxies were labeled "dusty" if patchy dust was obvious to the eye, or if the disk scale lengths in $\mathrm{U}$ and in $\mathrm{R}$ were very different, a consequence of dust extinction. Not surprisingly, the "dust-free" sample comprises fundamentally S0 and Sa galaxies.

\section{Results}

Colour gradients are present in most of the galaxies. While some positive gradients exist, negative gradients (bluer outward) are more common. Gradients in $B-R$ range from -0.25 to +0.15 magnitudes per decade in radius. Gradients in $U-R$ range from -0.4 to +0.1 , in the same units. We find a correlation between colour gradients and absolute magnitude of the bulge: larger bulges show negative gradients, while small bulges have positive gradients. Such relation cannot be a dust effect; when dusty galaxies are not excluded the trend changes sign, a consequence of the strong inward reddening in small dusty bulges. Bulge colours correlate with the absolute magnitude of the galaxy and the absolute magnitude of the bulge. All bulges with 


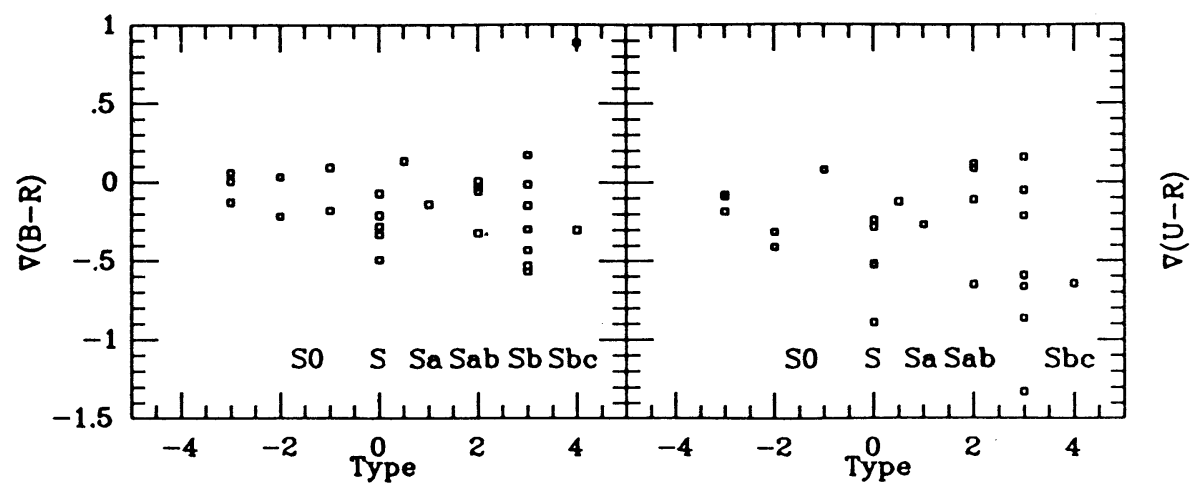

Fig. 1. left: $\nabla(B-R)$ as a function of galaxy type for the bulges sample. right: $\nabla(U-R)$ as a function of galaxy type. The increased dispersion in the gradients in suall bulges reflects the effects of dust reddening and star formation.

$B-R \geq 1.7$ turn out to be signifficantly affected by dust. The dust-free sample shows colours (at 5 ") ranging from $B-R=1.4$ to 1.65 . The bulge colours correlate well with the disk central colours, both for dusty and dust-free galaxies. However, the bulge colour gradients do not correlate with any disk photometric parameter.

Colour gradients in bulges and in ellipticals are similar, though not identical. The mean values for our dust-free bulges sample and the elliptical sample of Peletier et al. (1990) are essentially identical $(\langle B-R\rangle=-0.07$ and $\langle U-R\rangle=-0.20)$ For both ellipticals and bulges gradients become increasingly negative at absolute magnitudes $M_{B}$ between -18.5 and -21 . The relation gradient-luminosity is clcaner for bulges than it is for ellipticals. The main difference between ellipticals and bulges is the existence of positive colour gradients in small bulges, which is not seen in small ellipticals. Note however that the bulges sample extends to lower luminosities.

In summary, population gradients in early-type spiral bulges appear to be similar to population gradients in elliptical galaxies.

\section{Acknowledgements}

The Isaac Newton Telescope is operated on the island of La Palma by the Royal Greenwich Observatory in the Spanish Observatorio del Roque de los Muchachos of the Instituto de Astrofísica de Canarias.

\section{References}

Peletier, R. F., Davies, R. L., Illingworth, G. D., Davis, L. E. \& Cawson, M. 1990, Astron. J., $100,1091$.

Kent, S. M. 1986, Astron. J., 91, 1301. 